\title{
A model for assessing the systemic vulnerability in landslide prone areas
}

\author{
S. Pascale ${ }^{1}$, F. Sdao ${ }^{2}$, and A. Sole ${ }^{1}$ \\ ${ }^{1}$ Department of Environmental Engineering and Physics, University of Basilicata, Potenza, Italy \\ ${ }^{2}$ Department of Structures, Geotechnics, Engineering Geology, University of Basilicata, Potenza, Italy
}

Received: 1 March 2010 - Revised: 12 May 2010 - Accepted: 18 June 2010 - Published: 16 July 2010

\begin{abstract}
The objectives of spatial planning should include the definition and assessment of possible mitigation strategies regarding the effects of natural hazards on the surrounding territory. Unfortunately, however, there is often a lack of adequate tools to provide necessary support to the local bodies responsible for land management. This paper deals with the conception, the development and the validation of an integrated numerical model for assessing systemic vulnerability in complex and urbanized landslide-prone areas. The proposed model considers this vulnerability not as a characteristic of a particular element at risk, but as a peculiarity of a complex territorial system, in which the elements are reciprocally linked in a functional way. It is an index of the tendency of a given territorial element to suffer damage (usually of a functional kind) due to its interconnections with other elements of the same territorial system. The innovative nature of this work also lies in the formalization of a procedure based on a network of influences for an adequate assessment of such "systemic" vulnerability.

This approach can be used to obtain information which is useful, in any given situation of a territory hit by a landslide event, for the identification of the element which has suffered the most functional damage, ie the most "critical" element and the element which has the greatest repercussions on other elements of the system and thus a "decisive" role in the management of the emergency.
\end{abstract}

This model was developed within a GIS system through the following phases:

1. the topological characterization of the territorial system studied and the assessment of the scenarios in terms of spatial landslide hazard. A statistical method, based on neural networks was proposed for the assessment of landslide hazard;
2. the analysis of the direct consequences of a scenario event on the system;

3. the definition of the assessment model of systemic vulnerability in landslide-prone areas.

To highlight the potentialities of the proposed approach we have described a specific case study of landslide hazard in the local council area of Potenza.

\section{Introduction}

Landslide events can sometimes produce catastrophic effects on a given territory. Not only do they often cause loss of human lives but they can also cause serious damage to the supposed "vulnerable elements" of the territory, such as buildings or infrastructures, thus causing damage and temporary or permanent malfunctioning of economic and civic services and productive activities. This work puts forward a model for the assessment of vulnerability to landslide risk in inhabited areas, defined as Systemic Vulnerability, based on the application of a mathematical - decisional model which is able to assess the degree of landslide risk by analysing the destabilizing elements and the stress on the investigated system. The methodologies which have been utilized for the assessment of vulnerability in areas subject to landslide risk have not taken sufficiently into consideration the interconnecting functional relationships between the elements making up a territorial system.

One study which has looked at the functional relationships between interconnecting elements of a territorial system subject to natural hazards is Tamura et al. (2000), Ezell et al. (2000), Haimes and Jiang (2001), Minciardi et al. (2004) and Minciardi et al. (2006). 
The main aim of the proposed procedure is to act as a support in planning decisions, in analyses of the consequences of landslide events on complex territorial systems, in defining intervention priorities and in supplying quantitative and qualitative indicators to aid in the correct allocation of resources.

In other words the aim of the study is to assess the response to external stress (a natural calamity such as a landslide) in terms of the functional loss of the territorial system as a whole. Intervention elements which should be given priority are those with higher values of functional integrity $x_{i}$; moreover, the proposed procedure permits intervention on the elements which suffer the greatest systemic vulnerability effects.

The approaches mentioned above do not appear suitable for the analysis of the consequences of landslide events, because they deal with modelling aspects which are too specific. For the purposes of this work, a more general methodology is required so that the end user can make decisions regarding a determinate territorial system without being an export in modelling aspects of the particular type of risk involved.

For this reason a "systemic" approach to the analysis and assessment of vulnerability, based on a network of influences which takes into account the functional relationships existing among the elements of the territorial system has been developed.

Complex territorial systems are made up of several sub systems, with specific functions (e.g. inhabited settlements, health centres, environmental and civil protection structures, different infrastructures, energy distribution, etc.) which are (well or poorly) connected by causal and reciprocal relationships, and whose interactions are necessary to ensure a satisfactory and efficient functioning of the complex system.

In this regard, an assessment at the global level of the territorial system cannot be carried out without adequate models of the functional relationships existing among its sub systems. A system can become functional because of a direct influence on its elements or because the links existing between its sub systems become functional. For example, the collapse of a bridge, apart from the intrinsic loss, could bring about the interruption of an entire traffic zone; the putting out of service of a hospital building would have effects on the health system in general as well as follow on effects on mobility, communication, efficiency of civil protection forces, etc.

In overall terms, the efficiency of a territorial system cannot be defined as the sum of the vulnerabilities of its single components as the system itself has diverse functions (residential, industrial, etc.) which each involve several of its components. Moreover, these also generate follow on effects and so it is not only dependent on the number of vulnerable elements but also on the relevance each element has in the efficient functioning of the system.
Thus it is necessary to assess the vulnerability of a territorial system through careful analysis of the elements of the system and of their reciprocal relationships. This means that the concept of vulnerability shifts from being a characteristic of a particular territorial element to being a characteristic of a complex system whose elements are functionally related systemic vulnerability.

\section{Vulnerability: types and definitions}

With reference to instability phenomena vulnerability represents the level of loss produced in a given element or group of elements exposed to risk as a result of a natural phenomenon of a given intensity. It is expressed on a scale from 0 (no loss) to 1 (total loss) and is a function of the intensity of the phenomenon and the nature of the element at risk (Canuti and Casagli, 1996).

The introduction of the concept of vulnerability of territorial systems can be categorized as physical, functional and systemic vulnerability.

The term physical vulnerability represents the degree of loss of an element to suffer damage from external pressure (specifically from natural phenomena of a given intensity) (Canuti et al., 1999; UNESCO, 1979). The term functional vulnerability represents the tendency of an element to suffer impaired functioning due to external pressure (Fera, 1991; Di Gangi and Luongo, 2005). Systemic means the body as a whole, as well as the territory as a whole with people, infrastructures, industrial plant, natural elements, etc., and their interconnections.

The concept of systemic vulnerability (Minciardi et al., 2004, 2006; Fabietti, 1999; Khatibi, 2008) measures the tendency of a territorial element to suffer damage (usually functional) due to its interconnections with other elements of the same territorial system. It can be expressed as the analysis of the functional dependence of one element on the others.

Whilst physical and functional vulnerability may differ in relation to each other because of particular elements and circumstances, they are both dependent on the particular natural disaster (landslide, flood, earthquake) under consideration. On the other hand, the effects of systemic vulnerability on a territorial system are not linked to the particular disaster typology in question because they are related to the level of interconnections between the various elements making up the investigated territorial system.

\section{Assessment of vulnerability in complex territorial systems prone to landslide risk: the proposed model}

The proposed model, however, derived from a comprehensive bibliographical study of existing models used for other categories of natural disaster (Lagomarsino et al., 2002; Pascale et al., 2009; Minciardi et al., 2004, 2006) has been suitably modified and integrated so as to put forward a model applicable to landslide risk. 


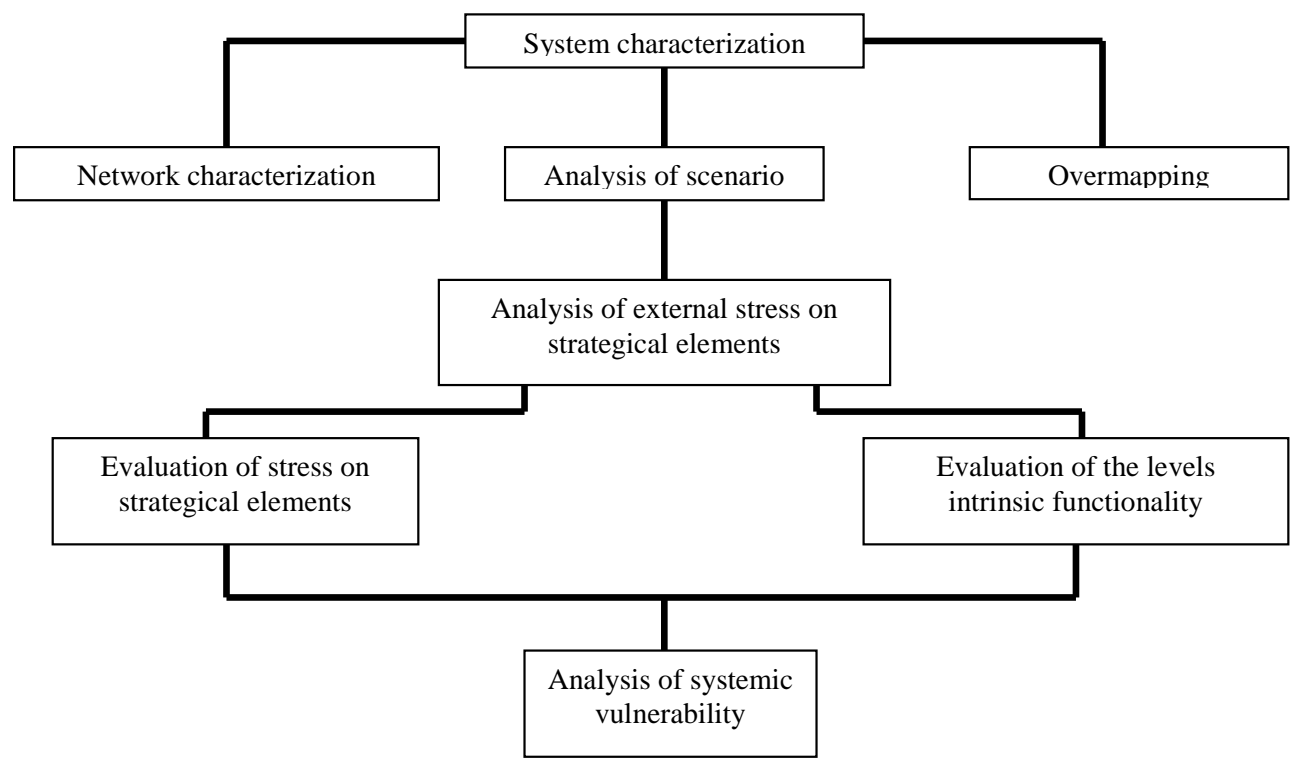

Fig. 1. Schematization of the main phases of the evaluation of systemic vulnerability.

The methodology utilized differs from that of other researchers in its implementation of a network rather than graphs (Minciardi et al., 2004, 2006) and diagrams of influence (Agogino et al., 1999; Howard et al., 1984; Schachter, 1988; Shenoy, 1992). The graph or diagram, are sequential line of relationship between elements, the network allows to reach a node from different lines. In particular, the influence network $G(E, A)$, is a graphic representation of the relationships between the variables involved in the study under consideration and it shows the flow of information, the existing influences, and the complex structure of the system. The network is useful in that it offers the possibility of understanding and codifying the conditional dependence between the elements of the territorial system without inserting probabilistic aspects.

In order to describe the proposed approach for the assessment of systemic vulnerability it is necessary to introduce specific notation. For each node, the members of the set $\mathrm{E}$ $(i=1, \ldots, N$, where $N$ is the number of territorial elements considered) the following variables are introduced:

- $\bar{\xi}_{i}^{\mathrm{k}}$ : vector of external solicitation relative to natural risk type $\mathrm{K}$ agents on the ${ }^{\prime} i$-th territorial element;

- $y_{i}$ : function of vulnerability of the $i$-th element with respect to landslide risk;

- $x_{i}^{0}$ : level of intrinsic functionality or the level of functional integrity of the element $i$-th, referred to physical integrity; $-x_{i}$ : level of functional integrity of the $i$-th element;

- $w_{i j}\left(x_{j}\right)$ : expresses the level of influence of the element $j$ on the level of functionality of the element $i$;

- $I_{\mathrm{a} ; \mathrm{b}}$ : influence between territorial elements;

- I: global Index I of systemic functionality.

Assessment of systemic vulnerability requires three phases:

1. characterization of the territorial system;

2. analysis of the direct consequences of a scenario event on the system under study;

3. focus on actual systemic vulnerability analysis (Fig. 1).

The procedure is implemented in a GIS system in such a way as to represent the elements/objects which make up the territory and contribute to the definition of vulnerability so as to make the territory dynamic and easily updatable.

The proposed model has been successfully applied on the territory of Potenza, which is well known for its widespread state of hydro-geological upheaval.

\subsection{Phase 1: Characterization of the system}

The first phase deals with the topological characterization of the territorial system and consists in the identification of its components on one hand and their connections and interactions on the other. 

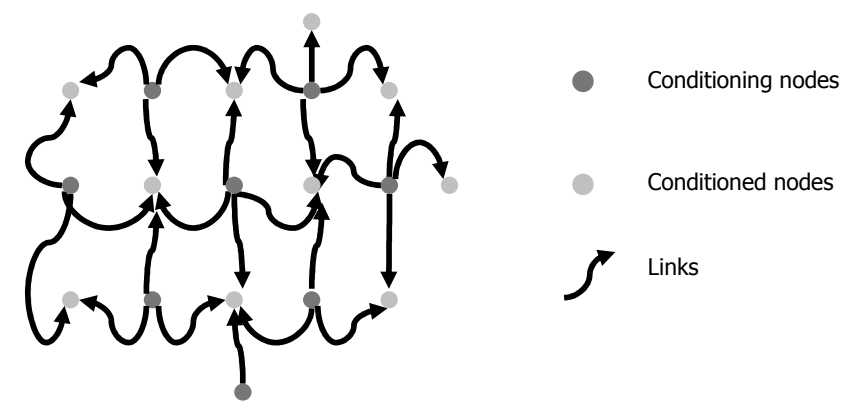

Fig. 2. Scheme of Influence network

The characterization of the system is itself made up of three phases: characterization of the network, scenario analysis, and overlay mapping of information.

\subsubsection{Characterization of the network}

The assessment of the entire functionality of a territorial system requires a consideration of the relationships existing within its subsystems, thus an analysis of the vulnerability of a territorial system requires not only an analysis of its single components but also of the relationships between them (Varis, 1997). In this way a territorial system is represented by a network G(E,A), (Pascale et al., 2007, 2009; Giosa et al., 2010; Gil and Steinbach, 2008) where E is the set of nodes and $\mathrm{A}$ is the set of links (Fig. 2). The nodes belonging to the set $\mathrm{E}$ represent all the relevant elements for the territorial system. The elements involved in the event are organized hierarchically according to their strategic importance (hospital, buildings, housing estate, railways, civil protection, police station, etc.) in line with the concept of "systemic vulnerability": the first element in the list is the most vulnerable node and so its break down could produce relevant effects in terms of both quantity and extension of damage to the system as a whole.

Damage to or malfunctioning of one of these elements may have a significant influence on the proper functioning of the entire territorial system. For example, a civil protection station may be inactive because a calamitous event has destroyed the building or because it has caused the collapse of a part of the transport network connecting it to the area involved in the event, thus impacting on the entire territorial system. Other examples are given in the work of Minciardi et al. $(2004,2006)$.

Such nodes can represent specific territorial locations (such as hospitals or police stations), linear elements (such as roadway infrastructures) or built-up areas (such as industrial areas, residential districts, dangerous or pollution sites, etc.). Three different, not necessarily distinct types of sets of nodes (Minciardi et al., 2006) have been identified:

1. set D of nodes representing the subsystems which, in response to external pressure, manifest a reduction in their physical and functional integrity and therefore require intervention from other subsystems; this category includes buildings (settlements), industrial areas, residential districts, etc.;

2. set $\mathrm{R}$ of the nodes representing the subsystems servicing the nodes which manifest reduced functionality; critical points such as hospitals, police stations, fire stations, civil protection, etc.;

3. set $\mathrm{S}$ of nodes representing infrastructural systems, which are fundamental in that they are often used to deliver services.

The links belonging to set $\mathrm{A}$, on the other hand, represent the relationships of influence between the functionalities of the territorial elements, i.e. between the diverse nodes. Specifically an element can be said to "influence" another element when the destruction or reduced efficiency of the first element has a negative effect on the functionality of the second. After an analysis of the area, of linear elements, and of key factors of the relationship between the identified elements is defined, for each pair of elements, an assessment is made of the existence of a reciprocal influence between the first and the second, lastly to each relation, (or arch of the network) is assigned one of four possible levels of importance (weak, medium-weak, medium-strong, strong).

\subsubsection{Analysis of scenario}

For the analysis and definition of the various scenarios, the reference point is the assessment of landslide hazard (Crozier and Glade, 2004), understood as spatial forecast (Canuti and Casagli, 1996).

In order to carry out the assessment of risk scenarios, the landslides hazard was determined through an innovative model obtained by means of Artificial Neural Network.

For the assessment of landslide susceptibility the inputs are represented by diverse parameters responsible for the instability of slopes which have been chosen on the basis of the geological and geo-morphological characteristics of the territory under study (slope angle, DEM, topographical index, lithology, land use, slope aspect and topographical shape) (Caniani et al., 2008; Ercanoglu, 2005), while the output is the landslide susceptibility map.

The map obtained from the ANN is subdivided into susceptibility classes varying from extremely high to low or zero to represent the final map. The areas of varying susceptibility represent the scenarios considered.

\subsubsection{Overlay mapping of the information}

The specific damage scenarios chosen which usually correspond to areas of high susceptibility to landslides were superimposed on the location map of the network elements in order to identify those elements which are directly involved in a possible damaging event. 
Table 1. Parameters and the relative scores utilized to calculate landslide magnitude.

\begin{tabular}{|c|c|c|c|c|c|c|c|}
\hline \multirow{2}{*}{$\begin{array}{l}\text { PARAMETER } \\
\text { Volume }\left(\mathrm{m}^{3}\right)\end{array}$} & \multicolumn{7}{|c|}{ RANGE OF VALUES } \\
\hline & "10 & $10-10^{2}$ & $10^{2}-10^{3}$ & $10^{3}-10^{4}$ & $10^{4}-10^{5}$ & $>10^{5}$ & \\
\hline Rating & 1 & 2 & 3 & 4 & 5 & 6 & \\
\hline Velocity (m/s) & $" 5 \times 10^{-10}$ & $5 \times 10^{-10}-5 \times 10^{-8}$ & $5 \times 10^{-8}-5 \times 10^{-6}$ & $5 \times 10^{-6}-5 \times 10^{-4}$ & $5 \times 10^{-4}-5 \times 10^{-2}$ & $5 \times 10^{-2}-5$ & $>5$ \\
\hline Rating & 1 & 2 & 3 & 4 & 5 & 6 & 7 \\
\hline Run out (km) & $" 10^{-3}$ & $10^{-3}-10^{-2}$ & $10^{-2}-10^{-1}$ & $10^{-1}-10^{-0}$ & $>10$ & & \\
\hline Rating & 1 & 2 & 3 & 4 & 5 & & \\
\hline Depth (m) & 1 & $1 \_12$ & $12 \_25$ & $25-35$ & $35-50$ & $>50$ & \\
\hline Rating & 1 & 2 & 3 & 4 & 5 & 6 & \\
\hline $\begin{array}{l}\text { Affected } \\
\text { area }\left(\mathrm{km}^{2}\right)\end{array}$ & “0.01 & $0.01-0.24$ & $0.25-0.50$ & $0.50-0.75$ & $0.75-1$ & $>1$ & \\
\hline Rating & 1 & 2 & 3 & 4 & 5 & 6 & \\
\hline Deformation & heterogeneous & homogeneous & continuous & discontinuous & & & \\
\hline Rating & 4 & 2 & 4 & 2 & & & \\
\hline Typology & $\begin{array}{l}\text { (Slowly) } \\
\text { Earth flow }\end{array}$ & $\begin{array}{l}\text { Roto-transla- } \\
\text { tional slides }\end{array}$ & Rock slides & $\begin{array}{l}\text { Rapid earth } \\
\text { flow }\end{array}$ & Rockfalls & Topples & Debris flow \\
\hline Rating & 2 & 4 & 8 & 8 & 10 & 10 & 10 \\
\hline
\end{tabular}

Table 2. Magnitude and intensity of the landslide.

\begin{tabular}{lcccccccccc}
\hline TOTAL RATING & 11 & $11 \_14$ & $14 \_18$ & $18 \_22$ & $22 \_26$ & $26 \_30$ & $30 \_34$ & $34 \_38$ & $38 \_42$ & 42 \\
\hline $\begin{array}{l}\text { MAGNITUDE } \\
\text { INTENSITY }\end{array}$ & I & II & III & IV & V & VI & VII & VIII & IX & X \\
\hline
\end{tabular}

(I) movement of a small area only perceived through extremely sophisticated instruments.

(X) violent movement of a large mass causing damage to several elements.

\subsection{Phase 2: Analyses of external stress on the elements}

In this phase, the elements which could be directly involved in a possible event are considered at each node, the elements belonging to the set $\mathrm{E}(i=1, \ldots N)$, where $N$ is the number of territorial elements considered:

$-\overline{\boldsymbol{\xi}}_{i}^{\mathrm{k}}$ : vector of external solicitation relative to natural risk type $\mathrm{K}$ agents on the ' $i$-th territorial element;

- $y_{i}$ : function of vulnerability of the $i$-th element with respect to landslide risk;

- $x_{i}^{0}$ : level of intrinsic functionality or the level of functional integrity of the element $i$ evaluated exclusively on the basis of its physical integrity. This term takes into account the physical vulnerability.

\subsubsection{Vector of external solicitation relative to natural risk type $K$ agent on the ' $i$-th territorial element $\left(\overline{\xi_{\mathbf{i}}}\right)$}

The components of the vector $\bar{\xi}_{i}^{\mathrm{k}}$ correspond to the physical quantities which characterize the intensity of the event $k$ for each element $i$. In a case of landslide risk these components could correspond to the magnitude or intensity of the landslide.

The magnitude should be defined as a function of landslide parameters. Because it is foreseen that the exact nature of the functional relationship between these landslide parameters can not be precisely defined, a relative scale rather than mathematical expression seems more appropriate. The set of parameters identified for better defining landslide magnitude are: volume, velocity, run out, depth involved area, deformation and typology. A possible method for combining the landslide parameters to devise a landslide magnitude scale is shown in Table 1. The method classifies each parameter in ranges and assigns a rating value to each category, then adds them to obtain a total rating. Ranges of rates are shown in Table 2 with each range assigned a value of landslide magnitude such as 1 to 10 . Finally, a correspondence between magnitude and intensity can be established as shown in the last row of the table for the two most extreme cases: minimum and maximum magnitudes. 


\subsubsection{Function of vulnerability of the $i$-th element with respect to landslide risk $\left(y_{\mathbf{i}}\right)$}

The function evaluates the degree of vulnerability of an entity when it is subjected to a stress resulting from a natural hazard process, therefore, it provides a quantitative assessment of the intensity of damage that a given stress, linked to the type of process $k$, can induce the item $i$.

Consider the simplest case where stress is seen as a simple scalar quantity. In such a case the vulnerability can be defined by a Gaussian curve corresponding to a mathematical function of an exponential type, known as Gaussian function, whose shape is shown in Eq. (1) (Giosa et al., 2010; Pascale et al., 2009).

The vulnerability of elements exposed to risk varying between 0 (no loss) and 1 (total loss), is calculated using the following equation:

$y=1-a \cdot \frac{e^{-\alpha \xi^{2,2}}}{\left(1+e^{-\alpha \xi^{2,2}}\right)}$

where: $\bar{\xi}_{i}^{\mathrm{k}}$ is the stress in relation to the considered risk; a is a constant which takes on a value equal to 2 and is calculated by fixing the boundary conditions $\left(\bar{\xi}_{i}^{\mathrm{k}}=0 e y=0\right.$, where $y=0$ represent $0 \%$ of vulnerability, i.e. no loss); $\alpha$ is a parameter calculated by fixing boundary conditions $\left(3<\bar{\xi}_{i}^{\mathrm{k}}<6\right.$; 0 , $3<y<1$ in a condition of medium to high vulnerability) and is equal to 0.02 .

Example 1: for $\bar{\xi}_{i}^{\mathrm{k}}=0$ and $y=0$

The Eq. (1) becomes

$0=1-a \cdot \frac{e^{-0}}{\left(1+e^{-0}\right)}=1-a \cdot \frac{1 / e^{0}}{\left(1+1 / e^{0}\right)}=1-a \cdot \frac{1 / 1}{(1+1 / 1)}=1-a \cdot \frac{1}{(2)}$

where $a=2$.

For the calculation of $\alpha$ we can consider that the value of indicator $\bar{\xi}_{i}^{\mathrm{k}}$ varies between 10 and 6 and is found between $y=1$ and $y=0.9$ in a condition of high vulnerability or we can consider that the value of indicator $\bar{\xi}_{i}^{\mathrm{k}}$ varies between 3 and 6 and is found between $y=0.9$ and $y=0.3$ in a condition of medium vulnerability.

Example 2: for $\bar{\xi}_{i}^{\mathrm{k}}=6$ and $y=0.45$

$0.45=1-2 \cdot \frac{e^{-\alpha 6^{2.2}}}{\left(1+e^{-\alpha 6^{2.2}}\right)}$

$0.45 \cdot\left(1+e^{-\alpha 6^{2.2}}\right)=1-2 \cdot e^{-\alpha 6^{2.2}}$

$0.45+0.45 e^{-\alpha 51.51}=1-2 \cdot e^{-\alpha 51.51}$

$0.45=1-2 e^{-\alpha 51.51}-0.45 e^{-\alpha 51.51}$

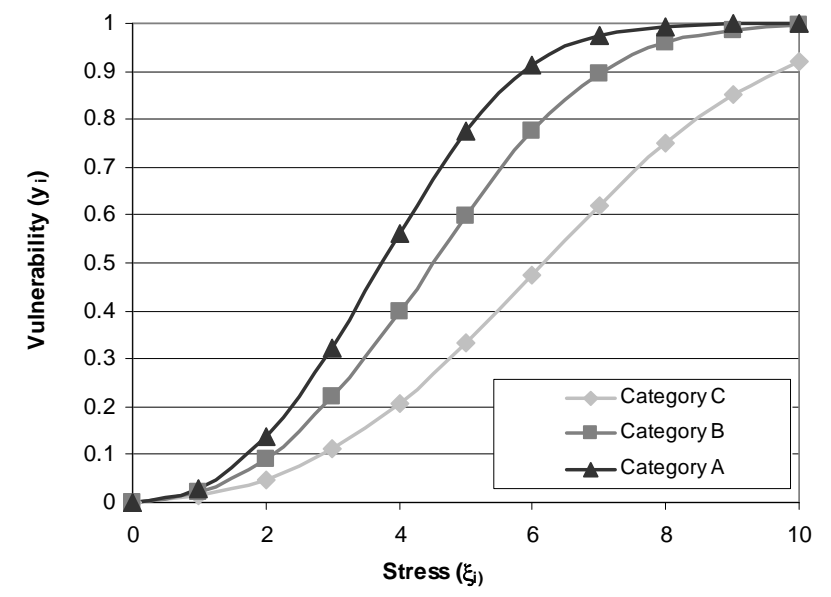

Fig. 3. Vulnerability curve for different types of risk elements. The categories A, B, and C are sets of risk elements with different physical, social and economic features.

$0.45=1-1.55 e^{-\alpha 51.51}$

$0.55=+1.55 e^{-\alpha 51.51}=1.55 \frac{1}{e^{51.51 \alpha}}$

$0.55=1.55 \cdot e^{-\alpha 0.0395}=1.55 \cdot \frac{1}{e^{0.0395 \alpha}}$

$e^{\alpha 51.51}=2.81$

$51.51 \alpha=\ln 2.81$

$\alpha=0.020$

Applying the Eq. (1) gives the curve of inherent vulnerability (Fig. 3); analytically it is justified by:

$\lim _{\xi \rightarrow 0} 1-a \frac{e^{-\alpha \xi^{2.2}}}{1+e^{-\alpha \xi^{2.2}}}=0$

$\lim _{\xi \rightarrow 1} 1-a \frac{e^{-\alpha \xi^{2.2}}}{1+e^{-\alpha \xi^{2.2}}}=1$

The role of this function is to assess the degree of vulnerability to which an element responds when subjected to landslide risk derived stress.

Vulnerable elements such as buildings or communication networks are subdivided into three categories A, B and C (Lagomarsino et al., 2002; Pascale et al., 2009; Thieken et al., 2008), where the categories A, B and C are sets of risk elements with different physical, social and economic features. The importance of these features moving from category $\mathrm{A}$ to $\mathrm{C}$, for example, different categories of buildings (rural stonework, reinforced concrete, steel, etc.) have different responses to stress. 
In this study it is assumed that $\alpha$ vary in the interval [0.01$0.1]$ defining in this way diverse vulnerability curves for diverse buildings and transport infrastructures types, (Fig. 5a, $\mathrm{b}$, and c) in particular:

- for $\alpha=0.06 \rightarrow$ to obtain vulnerability curve for category A elements;

- for $\alpha=0.04 \rightarrow$ to obtain vulnerability curve for category B elements;

- for $\alpha=0.02 \rightarrow$ to obtain vulnerability curve for category C elements.

Moreover the vulnerability curves were corrected as a function of population density (Eq. 2):

$y=1-a \cdot \frac{e^{-\alpha \xi^{(2,2+f)}}}{\left(1+e^{-\alpha \xi^{(2,2+f)}}\right)}$

where $f$ is the corrective factor assuming a value of 0 if population density is less than 5000 people $/ \mathrm{km}^{2}$ (source: ISTAT, 2001); 0.2 if the population density is between 5000 and 10000 people $/ \mathrm{km}^{2} ; 0.4$ if it is greater than 10000 people $/ \mathrm{km}^{2}$. Thus, in relation to population density, three different curves are obtained for each type of risk element studied (Fig. 4a, b, and c) (Pascale et al., 2009; Giosa et al., 2010).

\subsubsection{Intrinsic vulnerability $x_{\mathrm{i}}^{0}$ of the $i$-th node}

Intrinsic functionality describes the conditions of the territorial elements studied and is only and directly dependent on their physical vulnerability and does not consider any possible functional interconnection with other nodes or entities (Fera, 1991). The variable expressing the level of intrinsic functionality of an element $x_{i}^{0}$ can take on values within the interval between 0 and $1 ; 1$ indicates a low value of intrinsic functionality (element considered as outside the system) 0 indicates an optimal value of intrinsic functionality. The equation used to calculate intrinsic vulnerability is the following:

$x_{i}^{0}=\left(1-0.1 \tilde{\alpha}_{i}\right) \frac{\left(1-e^{-\tilde{\alpha}_{i} y_{i}^{2}}\right)}{\left(1-e^{-\tilde{\alpha}_{i}}\right)}+0.1 \tilde{\alpha}_{i}$

where

$y_{i}$ represents the value of vulnerability calculated in the $i$-th node of the Eq. (1);

$\tilde{\alpha}_{i}$ is a parameter which expresses the relation between the level of functionality $x_{i}^{0}$ of the node $i$ and the vulnerability $y_{i}$, i.e. it expresses the influence of the level of vulnerability $y_{i}$ of the element $i$ on its intrinsic functionality $x_{i}^{0}$; this parameter is assigned a weight of $(8,6,4$ or 2$)$ as a function of vulnerability $y_{i}$ :

- for $0.8<y_{i}<1 \tilde{\alpha}=8 \rightarrow$ extremely high intrinsic vulnerability;

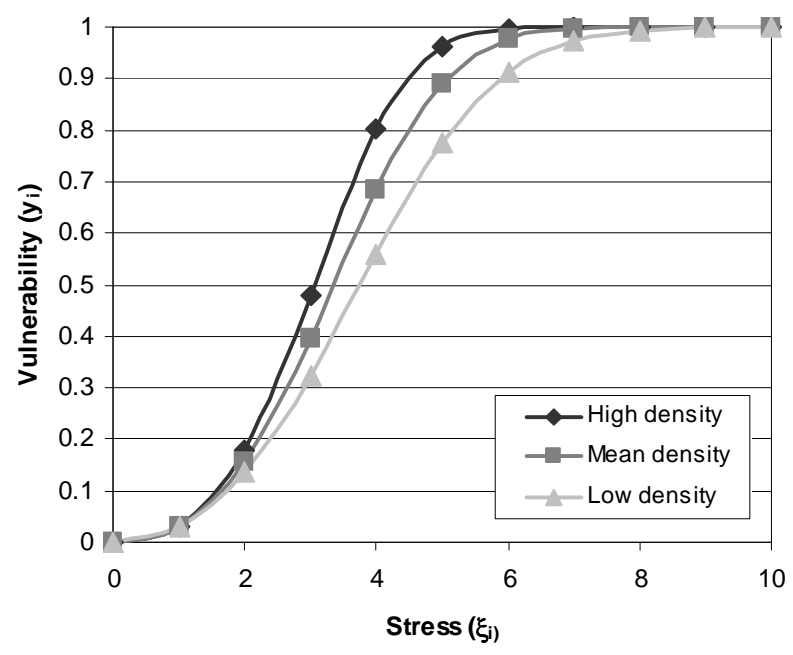

(a)

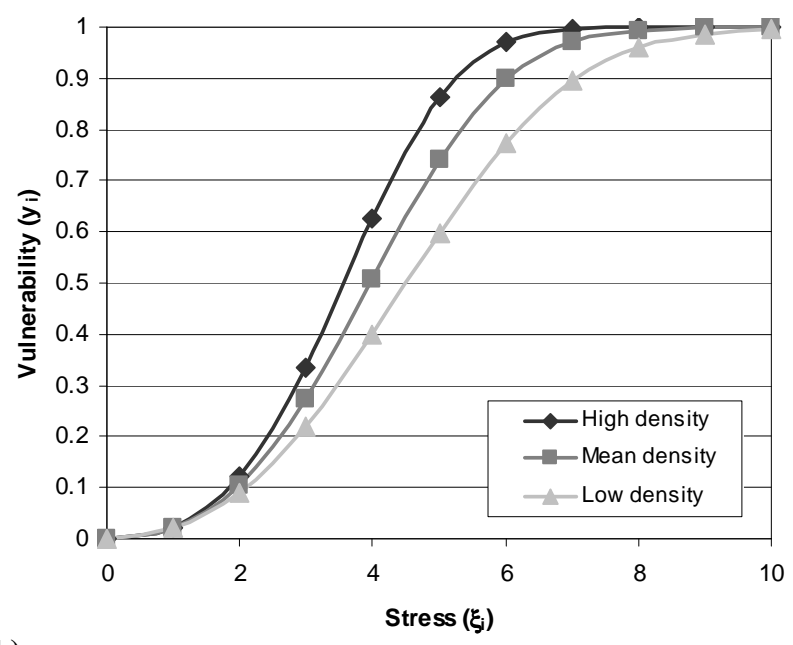

(b)

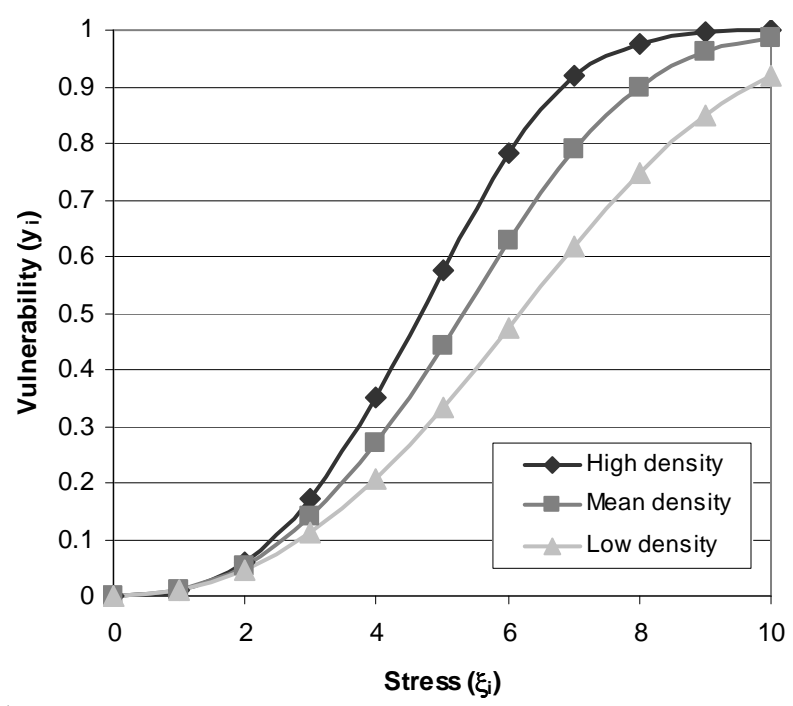

(c)

Fig. 4. Corrected vulnerability curves for category A (a), B (b), and C (c) elements using the Eq. (2). 


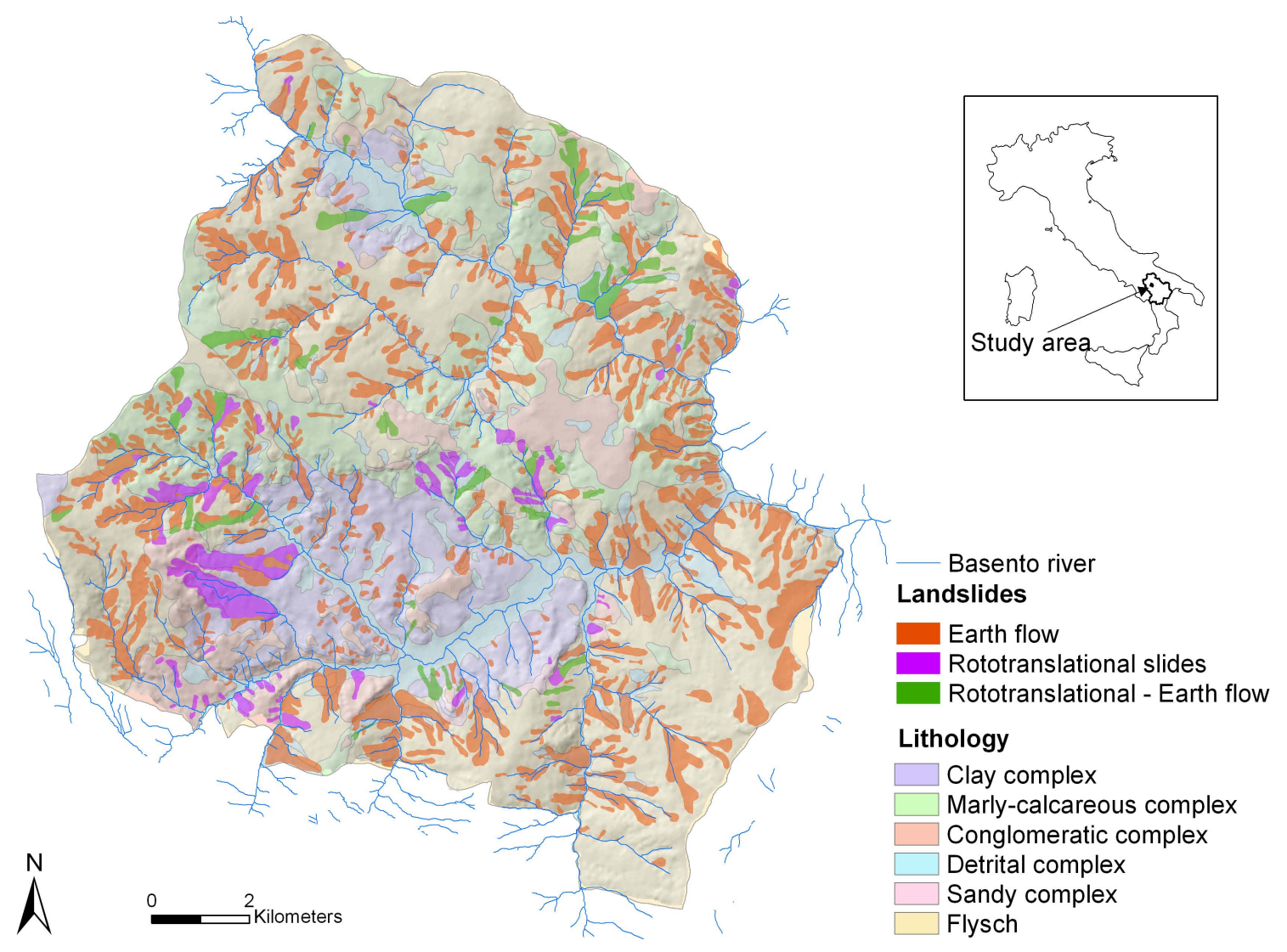

Fig. 5. Lithological and landslide inventory map of the study area (Caniani et al., 2008, mod.).

- for $0.6<y_{i}<0.8 \tilde{\alpha}=6 \rightarrow$ high intrinsic vulnerability;

- for $0.4<y_{i}<0.6 \tilde{\alpha}=4 \rightarrow$ medium intrinsic vulnerability;

- for $0<y_{i}<0.4 \tilde{\alpha}=2 \rightarrow$ low intrinsic vulnerability.

\subsection{Phase 3: Analyses of systemic vulnerability}

The third phase focuses on the actual systemic vulnerability analysis. Each node, falling within the risk scenario under consideration, is attributed a level of functional integrity $x_{i}$ which also takes account of its influence $w_{i j}$ on the various entities of the system studied. Following this, a global index of the entire system is obtained.

\subsubsection{Level of functional integrity $x_{\mathrm{i}}$ of the $i$-th element}

The level of functionality $x_{i}$ describes the conditions of functional integrity of the territorial element associated with the node $i$ involving information relative to the functioning of the other nodes. However, these conditions often do not coincide with the level of intrinsic functionality. The variable which expresses the level of functionality can also take on values which fall within the interval 0 and 1 , where 0 indicates the complete functional integrity and 1 corresponds to a state of total inoperativeness (poor functional integrity). It is reasonable to assume that the variable $x_{i}$ is dependent on both the level of intrinsic functionality of the considered element $x_{i}^{0}$ and the level of functionality of the elements which influence the functionality of $i$. The following equation is used to describe this dependence:

$x_{i}=\max \left(x_{i}^{0}, w_{i j}\left(x_{j}\right), \forall j \in P(i)\right)$

where the function $w_{i j}$ expresses the level of influence of the element $j$ on the level of functionality of the element $i$.

For example, if following a catastrophic event, a hospital does not suffer damage, but is connected by a single road that is destroyed, the hospital does not lose its intrinsic functionality, but its functional integrity.

It is possible to assume that the function $w_{i j}(x j)$, expressed through the use of a monotonous non decreasing function, characterized by range and co-range belonging to the interval $[0,1]$, can represent the influence of the functionality of the element $j$ on the functionality of the element $i$ through the following expression

$w_{i j}\left(x_{j}\right)=\left(1-0.1 \alpha_{i j}\right) \frac{\left(1-e^{-\alpha_{i j} x_{j}^{2}}\right)}{\left(1-e^{-\alpha_{i j}}\right)}+0.1 \alpha_{i j}$

where $\alpha_{i j}$ is a parameter which characterizes the arch, i.e. the relation between the node $i$ and the node $j$; note that when 
the value of $\alpha_{i j}$ increases, the level of influence of the functionality of the element $j$ on the functionality of the element $i$, decreases and vice versa.

The parameter $\alpha_{i j}$ is calculated through the relation $\alpha_{i j}=$ $-9.9^{*} I_{(\mathrm{a}, \mathrm{b})}+10$ where $I$, index of influence (see Sect. 3.3.2: index of influence $I_{(\mathrm{a}, \mathrm{b})}$ ), is the value of the influence calculated among the various risk elements.

On the basis of the obtained values of $\alpha_{i j}$, varying between 0 and 10 , it is possible to establish whether the influence between the two elements is weak, medium - weak, medium-strong or strong. The weak influence was assigned a value of $\alpha_{i j}<1$; the medium-weak influence was assigned $1<\alpha_{i j}<5$; the medium-strong influence was assigned $5<\alpha_{i j}<8$, and the strong influence was assigned a value of $\alpha_{i j}>8$.

\subsubsection{Index of influence $I_{(\mathrm{a}, \mathrm{b})}$}

The first step in the definition of the influence between diverse territorial elements consists in the determination of which category of elements is influenced by which other category of elements in an emergency phase. The main criteria on which this analysis is based is the assumption that the only categories able to exert any sort of influence are those belonging to the sets $\mathrm{R}$ and $\mathrm{S}$ of nodes which respectively represent service and infrastructure elements. The second assumption is that the elements belonging to $\mathrm{R}$ can only exert an influence on the elements belonging to set $\mathrm{D}$ (i.e. on the elements which expressly require services, in that they are directly involved in the event under consideration). Lastly, it is possible to hypothesize that the elements belonging to set $\mathrm{S}$ are able to exert an influence on all the elements belonging to the sets R U S U D (where $\mathbf{U}=$ union) (Table 3).

The second step is the definition of the level of such influences on the basis of objective information deriving from the analyses of the elements which make up the territorial system under examination. To these ends, on the basis of the work of Fiorucci et al. (2002), functions have been defined to supply the level of influence between the two categories for each pair of categories. In calculating the level of influence it has been hypothesized that the relation of supply and demand of services as well as the distance between the two elements considered play an important role in the definition of the level of dependence between them. Therefore for each couple of categories (a, b) an index of influence $I_{(\mathrm{b}, \mathrm{a})}$ was introduced and evaluated, these indexes take on values within the interval $[0,1]$. Table 4 shows the equations for the calculation of influence index defined for each couple of categories of territorial elements characterized by functional dependence.

For example, referring to the conditioning influence of a service center $\mathrm{p}$ of category $\mathrm{k}$ (e.g., such a category may include all civil protection centers and headquarters) over a urban settlement $r$, let us consider the following parameters:

- $\operatorname{Add}_{\mathrm{p}}^{\mathrm{k}}$ number of employees of center $\mathrm{p}$, of category k;
- $P_{i}$ number of inhabitants of urban settlement $\mathrm{r}$ involved in the event;

- $d_{\text {rp }}$ distance between urban settlement $r$ and center $\mathrm{p}$;

- $\operatorname{Add}_{\mathrm{q}}^{\mathrm{k}}$ number of technicians belonging to all the working units present in the system;

- $\mathrm{S}$ is the set of urban elements over the considered territory;

- $\mathrm{R}$ is the set of the critical points such as hospitals, police stations, fire stations, civil protection, etc.

- $\mathrm{O}$ is the sub-set of $\mathrm{R}$ that consists only of the operating units, without considering the health units.

A possible simple way to express the level of influence of element $p$ over element $r$ is that of introducing an Eq. (6):

$I_{i \mathrm{p}}^{\mathrm{k}}=\frac{\frac{\operatorname{Add}_{\mathrm{p}}^{\mathrm{k}}}{P i} \frac{1}{\sum_{\mathrm{q} \in \mathrm{R}} \operatorname{Add}_{\mathrm{q}}^{\mathrm{k}}} e^{-0.5 d_{\mathrm{rp}}}}{\max _{\mathrm{s} \in \mathrm{S}, \mathrm{q} \in \mathrm{O}}\left\{I_{\mathrm{sq}}^{\mathrm{k}}\right\}}$

Expressions analogous to Eq. (6) can be introduced for all the categories of elements that have to be taken into account in the vulnerability analysis of the territorial system.

\subsubsection{Global index $I$ of systemic functionality}

The final step in the proposed procedure is the calculation of the global index I of systemic functionality defined as the average value of functional integrity of all the elements considered and can be expressed as

$I=\frac{\sum_{i=1}^{N} x_{i}}{N}$

This index expresses the value of functional integrity of all the elements considered and it also varies within the interval $[0,1]$ where 0 indicates no functional loss, and 1 total functional loss.

Following this, Table 5 was elaborated as a function of the parameter $I$ where the functionality index was subdivided into opportune intervals, each of which was assigned a value of functional loss varying between low and high.

\section{Application to a case study: municipal area of Potenza (Basilicata, Southern Italy)}

Geological, geo-morphological, climatic and seismological factors make Basilicata (Southern Italy) one of the Mediterranean regions at risk due to high-magnitude geomorphological phenomena. This region is sensitive to extensive and severe slope movements. A variety of triggering mechanisms come into play, namely frequent earthquakes 
Table 3. Identification of the influences existing between the diverse categories of territorial elements, where the category reported on a particular line is influenced by the category reported in a specific column the corresponding box reports a V, while it reports an $\mathrm{X}$ in the opposite instance.

\begin{tabular}{lccccc}
\hline Conditioned nodes/ & & & & & \\
Conditioning nodes & Settlements & Route links & Headquarters & Health units & Industrial plant \\
\hline Settlements & $\mathrm{X}$ & $\mathrm{X}$ & $\vee$ & $\vee$ & $\mathrm{X}$ \\
Route links & $\vee$ & $\vee$ & $\vee$ & $\vee$ & $\vee$ \\
Headquarters & $\vee$ & $\vee$ & $\vee$ & $\vee$ & $\mathrm{X}$ \\
Health units & $\vee$ & $\mathrm{X}$ & $\vee$ & $\vee$ & $\mathrm{X}$ \\
Industrial plant & $\mathrm{X}$ & $\mathrm{X}$ & $\vee$ & $\vee$ & $\mathrm{X}$ \\
\hline
\end{tabular}

(VIII to IX MCS), periodic heavy rainfall and floods (one event every 6 months in the last 80 years) and reckless human behaviour. Over the last few decades, some large and recurrent landslides have affected urbanized areas, causing severe damage to properties. Sometimes such landslides have happened even after not excessively heavy rainfall (Sdao et al., 1996; Polemio and Sdao, 1996, 1998, 1999; D’Ecclesiis et al., 1991).

The proposed procedure for the estimation of systemic vulnerability in urban areas with high landslide risk was applied in the municipality of Potenza. This area has a well known and widespread susceptibility to landslides which have often caused serious damage to structures and transport infrastructures.

\subsection{Geological framework}

The municipal area of Potenza was selected as suitable for the evaluation of susceptibility to landslides mainly because it is typical of the geo-morphological and geological settings of landslide processes. The municipality Potenza is located in Southern Italy. It covers approximately $174 \mathrm{~km}^{2}$. The area is a part of the Apennine Chain and has a average annual rainfall of about $800 \mathrm{~mm}$. Elevation ranges from 593 to 1339 m a.s.l.

The geological framework of the municipal area of Potenza is characterized by a Plio-Pleistocene clastic succession which overlies, in an irregular manner, the MesoCenozoic flysch-type clayey-marly successions, belonging to the Lagonegro and Sicilide Units (the latter also known as Varicoloured Clays (Di Nocera et al., 1988). The Lagonegro and Sicilide Units were structured in a fold-and-thrust system during the Oligo-Miocene orogenic phases. The Pliocene successions belong both to the Altavilla Unit, characterized by a conglomeratic-arenitic succession which outcrops along the edges of the Potenza basin (e.g. in localities Contrada Botte - Poggi di S. Michele), and to the Ariano Unit, which is made up of a succession of conglomerates, arenites and clays with a marked lateral variability. These successions were deposited in intra-Apennine basins during the late orogenic phases (Upper Messinian-Pliocene), and later involved in intense tectonic phases with a prevalently vertical component from Upper Pliocene to Quaternary, which have determined the present shape of the Apennine Chain (Fig. 5).

\subsection{Landslides}

As noted above, most of the territory of Potenza is characterized by structurally complex geological substratum which is particularly prone to slope instability phenomena because of its structural and lithological characteristics. Recent studies have shown that, in this area, most slopes show a tendency to landslides of various types and dimensions, many of which are active and periodically cause serious widespread damage to things and sometimes to people. The methodological approach for the definition of the state of the landslide activity in the area of Potenza took advantage of the synergy between the methods of applied and evolutive geomorphology, traditional techniques for the recognition of landslides (aerial photo analyses and cartographic elaborations) and geo-morphological surveys. Studies have also taken into consideration the results of previous research regarding the area under study and its bordering areas. Moreover, in order to delineate a complete landslide inventory map of the area under study, a considerable amount of data was utilized: accurate geological and geo-morphological surveys, scale $1: 2000$ or 1:5000, carried out in 1998, 1999, and 2004; geognostic, stratigraphic and inclinometric data related to many boreholes carried out in the study area and in correspondence with the main body of recognized landslides; a comparative analysis of aerial photography of diverse periods and scales was made.

The present situation of slope instability of the examined areas is illustrated on a landslide inventory map (Fig. 5) drawn up for this purpose, on a 1:5000 scale, which shows, for every indicated landslide, and movement typology, the state of activity and the direction of movement. A total number of 920 landslides, covering an area of approximately $46 \mathrm{~km}^{2}$, which corresponds to $26 \%$ of the entire municipal area of Potenza, were recognized in the study area. The characteristics of landslide movements vary from rotational slides to rapid earth-flows with different phases of 


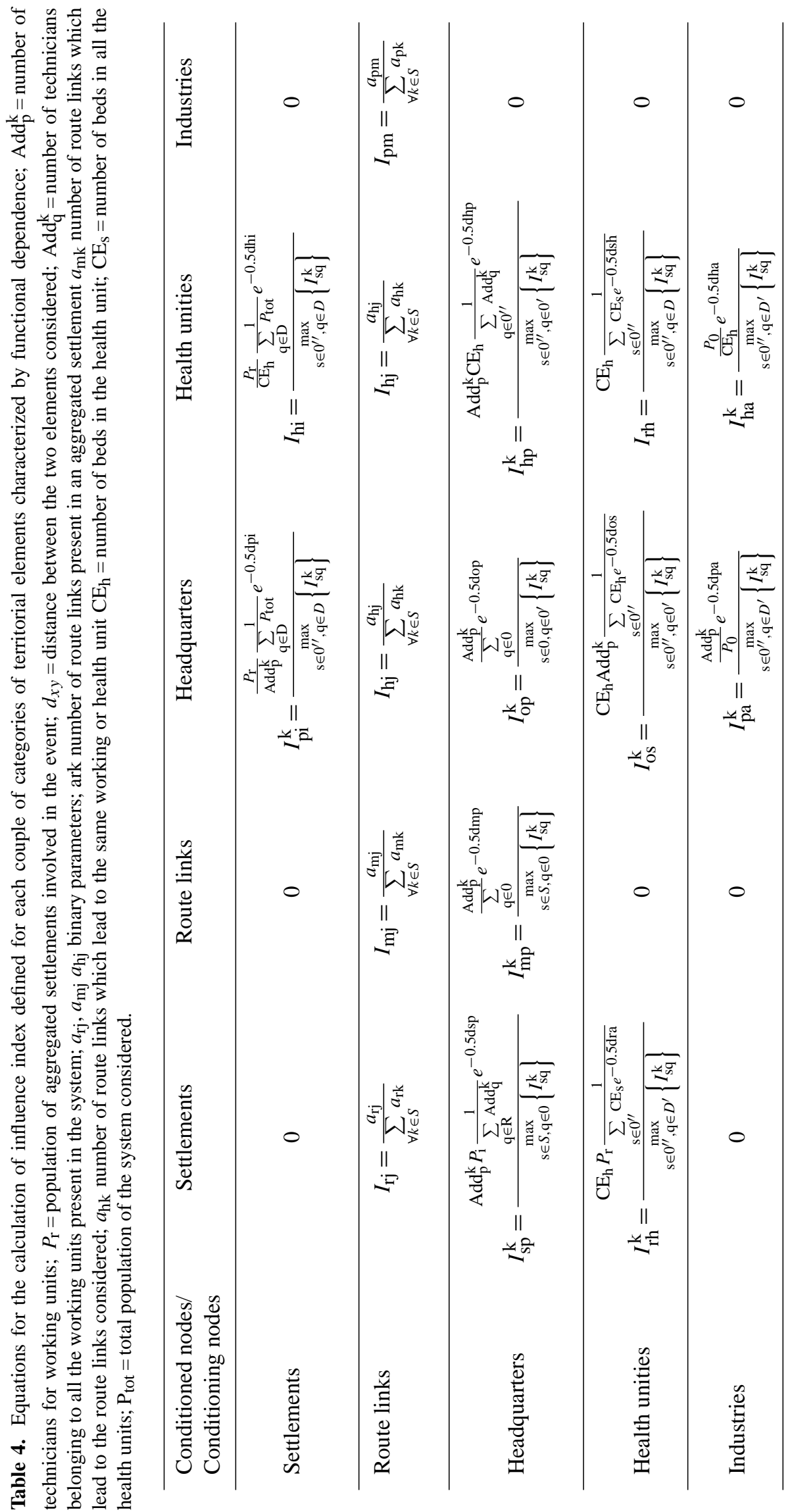


Table 5. Functionality index and respective functional loss.

\begin{tabular}{lcccc}
\hline Functionality global index $I$ of system & $0-0.25$ & $0.25-0.5$ & $0.5-0.75$ & $0.75-1$ \\
\hline Loss of functionality & Low & Medium & High & Very high \\
\hline
\end{tabular}

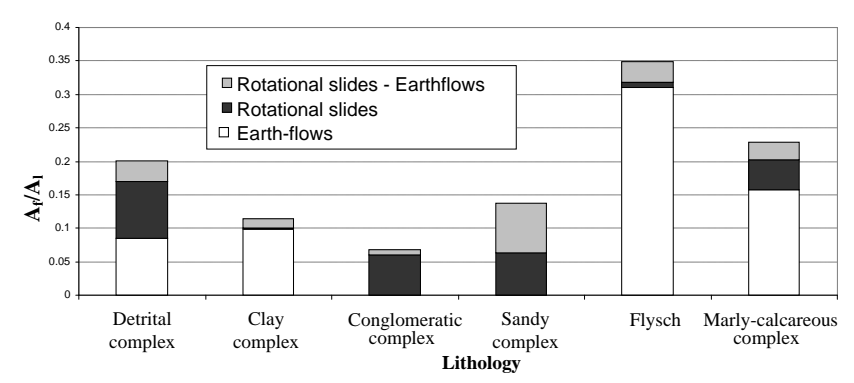

Fig. 6. Landslide distribution according to lithological class (Caniani et al., 2008).

movement. It is worth noting that many of the landslides are quite thick, more than 15-20 m, and that the earth-flows are the most common. The graph in Fig. 6 shows the landslide distribution for each lithological class. The typology and the geometric and evolutive characteristics of the landslides are strongly influenced by the lithological and structural characteristics of the outcropping terrains. Where the outcrops are clastic, conglomeratic-sandy-clay, dating to the Pliocene age, landslides have a rotational or multiple retrogressive roto-translational character, in correspondence with extensive outcrops of marly-clay terrain belonging to Varicoloured Clay. Flysch Galestrino landslides are extremely widespread as earth-flows fed by roto-translational slides present in the high parts of the slope.

\subsection{Characterization of the territorial system of the city of Potenza}

Potenza has 96000 inhabitants and it has a complex territorial system characterized by several critical factors related to important elements at risk. Near the urbanized centre of Potenza there are industrial plant, railways, main and local roads, health and professional centres, a large concentration of residential and commercial areas and productive activities.

\subsubsection{Network characterization}

The network, representing the territorial system, has 18883 elements (Fig. 7) with 1283 areal elements (residential districts), 5000 lines (infrastructures: streets, roads, railways and so on) and 21000 points (crossroads, public buildings such as schools, local health centres, hospitals, military and fire stations, industrial plant and so on).
A relational structure was defined between the identified elements (points, lines and areas) which is determined by which categories of elements are influenced by which other categories of elements in an emergency phase. Firstly the existence of a relationship between two elements was verified. Then for each for each couple of elements in relationship, an assessment of the influence between them was carried out (territorial influence) through an index of influence $I_{\mathrm{b}, \mathrm{a}}$ (Table 1) as described in Sect. 3.3.2; Tables 3 and 4.

\subsubsection{Scenario analysis}

The landslide scenario considered in this work involves the locality "Varco d'Izzo" in the district of Potenza (Fig. 8). It is an area which has been recently subject to extensive urbanization and is marked by the diffuse presence of human activity and several important transport routes (S.S. 407 Basentana, Railway Potenza-Metaponto). The city of Potenza is on one of the most important rivers of the Basilicata, the Basento; the left slope of the Basento River, on which the eastern portion of Potenza extends, is strongly affected by large and old landslides which are represented by huge rototraslational slides evolving to earth-flows. These landslides are generally quiescent but they are prone to more or less significant phases of reactivation. The landslide scarps which characterize the higher portions of the slopes are wide and generally depleted. The accumulation zones of the landslides are clearly visible and they are subject to periodic remobilization phases. These zones are generally fan-shaped and their toes reach the Basento channel which consequently is meandered with evident lateral deviations.

These widespread landslide phenomena are often reactivated, especially due to earthquakes and rainfall, causing serious damages to both the buildings and the roadways of the area.

One of the largest mass movements in this area is represented by a very old slide - earth flow (scenario of the area study) involving the whole slope of Varco d'Izzo (Fig. 8), which has been recently affected by widespread urbanization (indeed, on 1956 aerial photos this area was almost uninhabited and without any infrastructure), and whose toe is now close to the two main lines of communication of this part of the region: the National Road 407 Basentana and the Railway Potenza - Metaponto. In Table 6 the morphometric features of the slide - earth flow are illustrated (Perrone et al., 2004). 


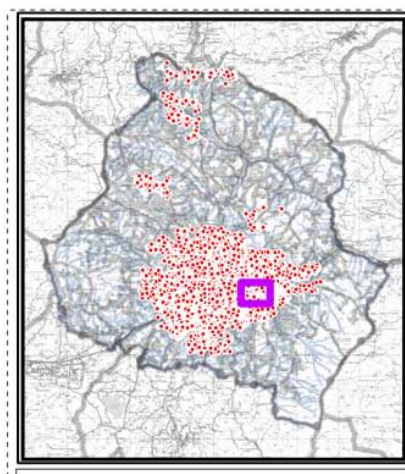

Legend

- Point elements

- Road network

$X$ Crossroads

$\sum$ Census area

- River network

Reference System: Gauss Boaga, Roma 40 fuso EST

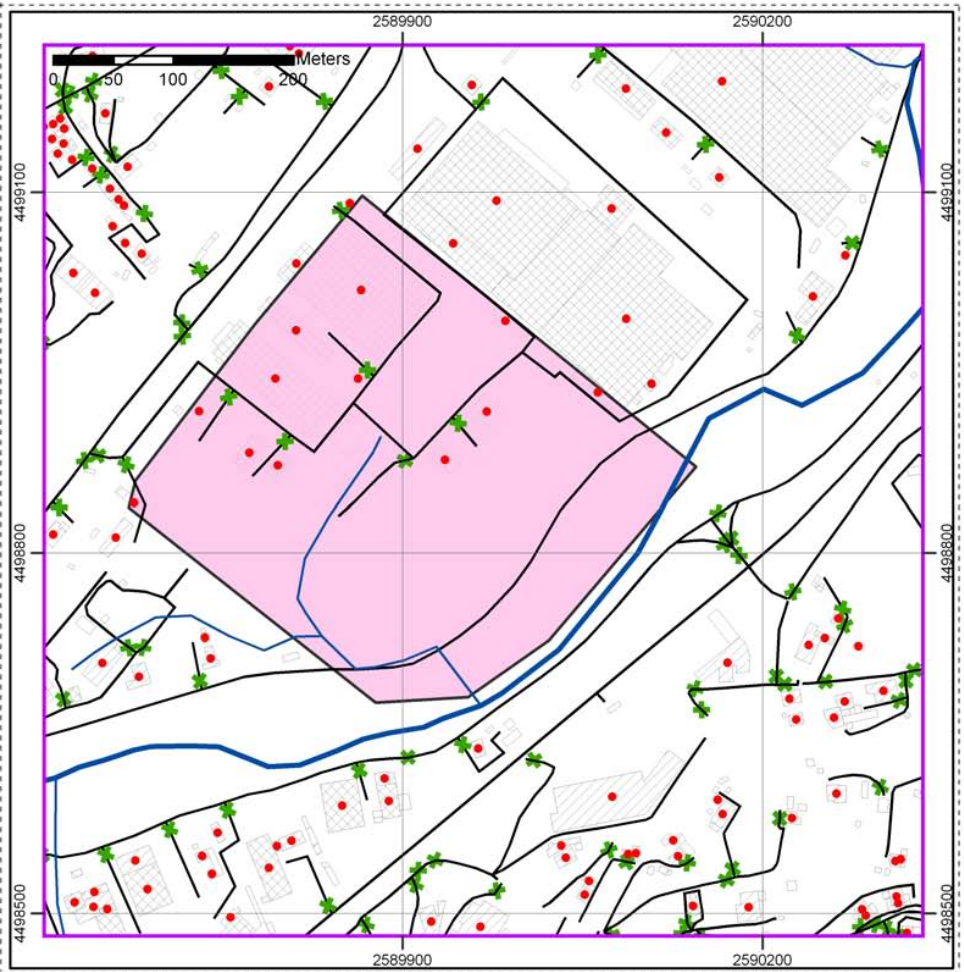

Fig. 7. Network characterization with the linear links among the elements that form the network. The connections may have different combinations of elements of various types: areas with areas, points with points or areas with points.
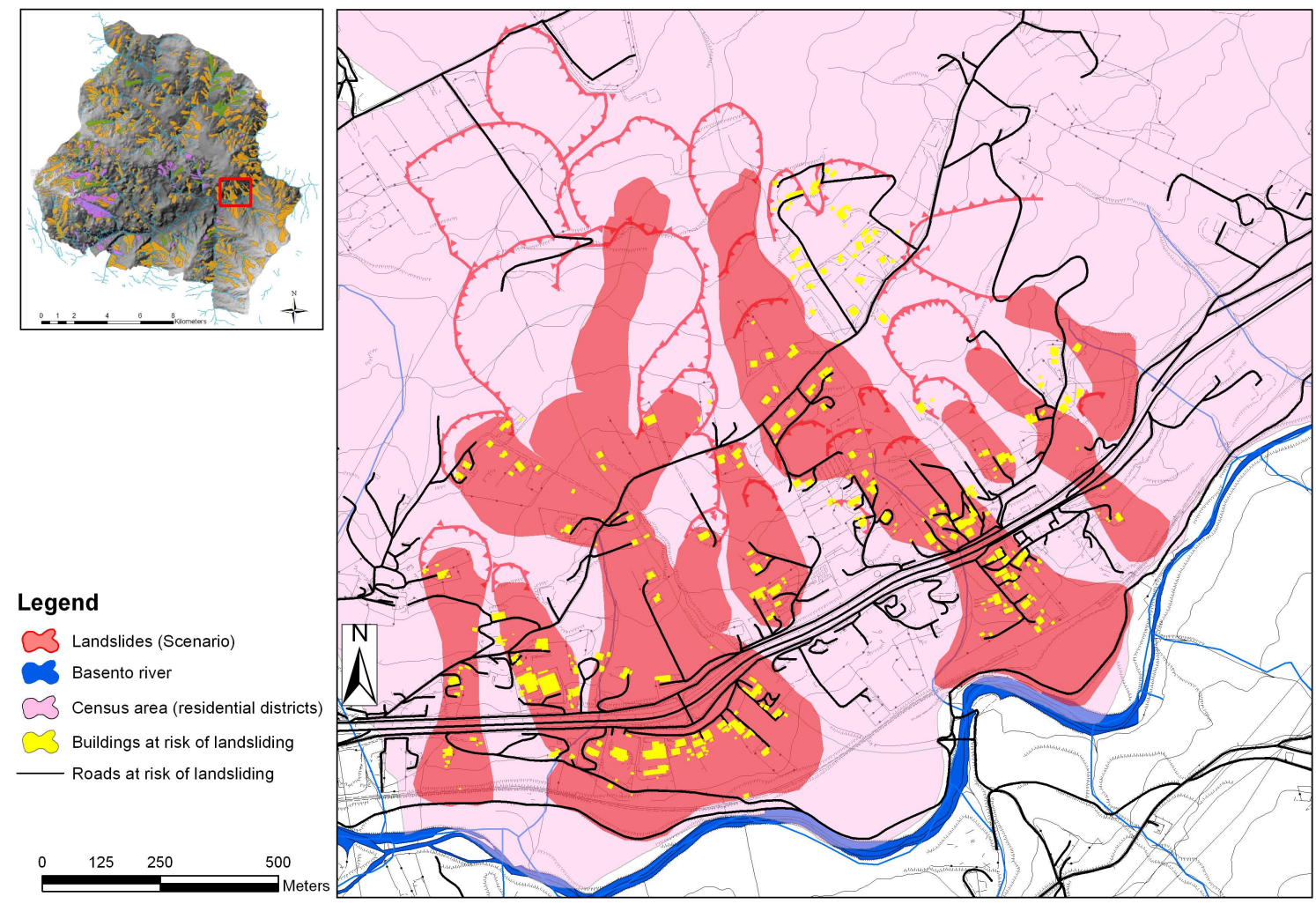

Fig. 8. Potenza city data overmapping with landslide area and elements directly involved by landslide. 


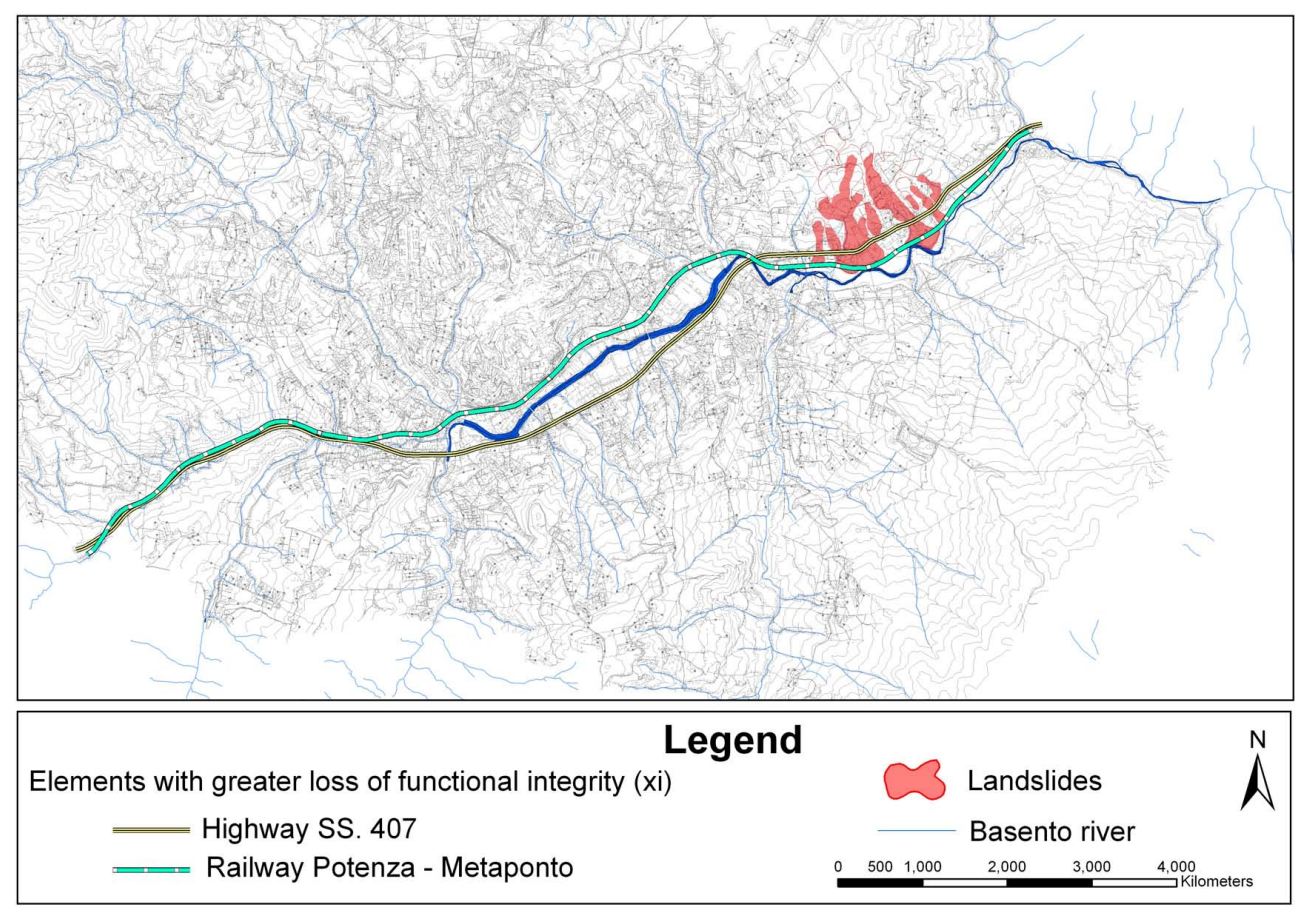

Fig. 9. Potenza city and element with greater loss of functional integrity $\left(x_{i}\right)$.

Table 6. Morphometric features of the flow - earth flow of Varco d'Izzo.

\begin{tabular}{lcccc}
\hline & Length $(\mathrm{m})$ & width $(\mathrm{m})$ & Inclination $\left(^{\circ}\right)$ & Thickness max $(\mathrm{m})$ \\
\hline Source area & 300 & $100-200$ & 16 & $25-30$ \\
Flow track & 700 & $150-250$ & 13 & 20 \\
Zone of accumulation & 350 & 500 & 8 & $20-34$ \\
\hline
\end{tabular}

Table 7. Physical vulnerability and intrinsic functionality for some characteristic elements of the study.

\begin{tabular}{lccc}
\hline Type & Category & Vulnerability phisical & Intrinsic functionality \\
\hline Roads & A & 0.89 & 0.99 \\
Residential district or census areas & B & 0.74 & 0.98 \\
\hline
\end{tabular}

\subsection{Data overmapping}

The scenario has been superimposed on the map of the elements of the system (Fig. 8). Thus, the elements that are directly affected by the disaster are known: 226 road links, 418 inhabited settlements; the latter including the smallest (single buildings) and the largest (conurbations).

\subsection{Systemic vulnerability assessment}

The analysis of systemic vulnerability of the territorial system under study was carried out for the selected scenario (see Sect. 4.3 and Fig. 8). The results were obtained through the application of the procedure proposed in Sect. 3 .
The scenario utilized (Fig. 8), 26 territorial elements directly involved in the disaster: 6 areas, 6 points ( 5 buildings and 1 industry) and 20 lines. The following evaluations were made for each of these elements: physical vulnerability $\left(y_{i}\right)$ and intrinsic functionality $\left(x_{i}^{0}\right)$ using the Eqs. (2) and (3) (Pascale et al., 2009; Giosa et al., 2010). Table 7 shows the values of physical vulnerability and intrinsic functionality calculated for some characteristic elements of the study.

In the case studied, physical vulnerability varies from 0.44 to 0.89 for the linear elements and 0.74 for the areas. Intrinsic functionality varies from 0.72 to 0.99 for the linear elements and 0.98 for the areas. 
For the scenario under consideration, in systemic vulnerabilities, the input data comes from the values of intrinsic functionality of the spatial elements in the system.

Below, $x_{i \mathrm{~T}}, x_{i \mathrm{~T}}^{0}$ and $I_{\mathrm{T}}$ are the level of functional integrity, the level of intrinsic functionality and the global index of functionality for the $i$-th element.

All the considered elements show a functional loss of at least $10 \%$ and, therefore, not negligible.

All the linear elements have a loss of functional integrity greater than 20\%: these are urban streets, subways and railways (close or directly involved in the area of risk) and, therefore, with null values of both the functionality and the intrinsic functionality.

Six roads have more intrinsic functionality $\left(x_{i}^{0}\right)$ and functional integrity $\left(x_{i}\right)$ reduction (Fig. 9).

This reduction depends on the low value of functionality of the streets and it is not due to a loss of intrinsic functionality, but rather to a loss of functional integrity of the roads connected to them.

The procedure of analysis of the systemic vulnerability was also used to identify the element (or elements) that, in relation to the scene considered, greatly influences the functionality of the territorial system which is specific to the case of National Road 407 Basentana and of the railway line Naples-Taranto.

The global index of functionality loss is 0.87. Overall, for the selected scenario, the landslide in the locality "Varco d'Izzo" in the city of Potenza, the loss of functionality for the entire territorial system making up the territory under study, was found to be extremely high.

\section{Conclusions}

This work has put forward an approach for the estimation of the consequences of landslide events drawn up by means of the utilization of a territorial model based on a network of influences and an estimation procedure of systemic vulnerability.

The concept of vulnerability is no longer considered simply as the characteristic of a single element but as associated with a complex territorial system of fundamental importance.

The reliability of the proposed approach was tested on a case study regarding landslide risk in the district of Potenza (Basilicata, Southern Italy).

The infrastructural elements that mainly affect the functionality of the territorial system have been identified in the National Road 407 Basentana and the Railway NaplesTaranto. Although the same conclusion could also have been achieved by the analysis carried out by an expert in the territory (due to the relative simplicity of the territorial system considered), it is to be considered as an important result, because it highlights the reliability of the obtained results and, consequently, of the decisions to come.
The significance of such analyses in the ambit of the particular territory considered must also be emphasized. Analogous events to those considered in the study have occurred in the Potenza area and have caused serious damage to the main infrastructural systems (water pipelines and roadways) as well as direct and indirect economic losses on most of the surrounding area. On the basis of these considerations, it would seem appropriate to utilize tools capable of carrying out adequate territorial analyses, including those from a systemic perspective, which are able to furnish reliable and usable results for territorial planning and for an improved definition of emergency management strategies.

The analytic procedure of systemic vulnerability appears highly applicable to complex territorial systems in terms of the dimensions of the area considered as well as the number of territorial elements directly involved in the phases of the immediate aftermath of the emergency because services are required and need to be supplied.

This procedure can be used to identify the functionality of a territorial system subject to landslide risk due to the existence of functional links between diverse elements or to determine the lack of appropriate interventions (structural or management) in the territorial system. Therefore, the goal of the model is the identification of the areas of greater vulnerability within the urban fabric and as an aid in the definition and programming of urban strategies against landslide risk.

At this stage, the study, which is still in progress, provides the extension of the proposed model to cases where the vulnerability is affected by combined natural phenomena. This occurs, for example, when different natural events are triggered at the same time, or are linked to each other. For instance, landslides may obstruct the regular stream flow and produce a dam breaking effect downstream.

Acknowledgements. We are grateful to Elisabetta Barletta and Marcello Bianca for the fruitful discussion.

Edited by: T. Glade

Reviewed by: M. Keiler and another anonymous referee

\section{References}

Agogino, A. M.: Management of uncertainty with influence diagrams, University California in Berkeley, working paper 850703-6, 1999.

Caniani, D., Pascale, S., Sdao, F., and Sole, A.: Neural networks and landslide susceptibility: a case study of the urban area of Potenza, Nat. Hazards, Springer (Ed.), 45, 55-72, 2008.

Canuti, P. and Casagli, N.: Considerazioni sulla valutazione del rischio di frana, estratto da "Fenomeni franosi e centri abitati", Atti del Convegno di Bologna del 27 Maggio 1994, CNR - GNDCI Regione Emilia Romagna, 57 pp., 1996.

Canuti, P., Casagli, N., Catani, F., and Fanti, R.: Hydrogeological hazard and risk in archaeological sites: some case studies in Italy, J. Cult. Herit., 1, 117-125, 2000. 
Crozier, M. J. and Glade, T.: Landslide Hazard and Risk: Issues, Concepts and Approach, in: Landslide risk assessment, edited by: Glade, T., Anderson, M. G., and Crozier, M. J., John Wiley, 1-40, 2004.

D'Ecclesiis, G., Grassi, D., Merenda, L., Polemio, M., and Sdao, F.: Evoluzione geomorfologica di un' area suburbana di Castronuovo S. Andrea (PZ) ed incidenza delle piogge su alcuni movimenti di massa, Geologia Applicata e Idrogeologia 26, 141-163, 1991 (in Italian).

Di Gangi, M. and Luongo, A. S.: Vulnerabilità di un sistema di trasporto in condizioni di emergenza: indicatori quantitativi per l'analisi in Modelli e metodi per l'analisi delle reti di trasporto in condizioni di emergenza: contributi metodologici ed applicativi (a cura) di Massimo Di Gangi, Ermes, 114-126, 2005 (in Italian).

Ercanoglu, M.: Landslide susceptibility assessment of SE Bartin (West Black Sea region, Turkey) by artificial neural networks, Nat. Hazards Earth Syst. Sci., 5, 979-992, doi:10.5194/nhess-5979-2005, 2005.

Ezell, B. C., Farr, J. V., and Wiese, I.: Infrastructure risk analysis model, J. Infrastruct. Syst., 114-117, 2000.

Fabietti, W.: Vulnerabilità e trasformazione dello spazio urbano, ALINEA Editrice, Firenze, 373 pp., 1999.

Fera, G.: La città antisismica, Gangemi Editore, Roma, 1991 (in Italian).

Gil, J. and Steinbach, P.: From flood risk to indirect flood impact: evaluation o street network performance for effective management, response and repair, First International Conference on Flood Recovery, Innovation and Response, 335-344, 2008.

Giosa, L., Pascale, S., Sdao, F., Sole, A., and Albano, R.: A systemic approach to analyze territorial vulnerability during flood events, Journal of Flood Risk Management, in press, 2010.

Haimes, Y. Y. and Jiang, P.: Leontief-based model of risk in complex interconnected infrastructures, J. Infrastruct. Syst., 1-12, 2001.

Howard, R. A. and Matheson, J. E.: The principles and applications of decision analysis, Strategic Decisions Group, Palo Alto, CA, 719-762, 1984.

Khatibi, R.: Systemic nature of, and diversification in system exposed to, flood risk, First International Conference on Flood Recovery, Innovation and Response, 91-101, 2008.

Lagomarsino, S., Giovinazzi, S., and Lazzoni, L.: Il rischio sismico: valutazione della pericolosità, analisi della vulnerabilità fisica del costruito e scenari di danno, dossier tematico - Progetto GERIA (Gestione dei Rischi Ambientali), Programma UE INTERREG II a, per la cooperazione transfrontaliera Italia - Francia, collana Territorio e Ambiente, Edizione del Delfino Moro, Alberga, 2002.
Minciardi, R., Sacile, R., Taramasso, A. C., Trasforini, E., and Traverso, S.: Valutazione della vulnerabilità di sistemi territoriali complessi soggetti a rischi di origine naturale: il caso della sollecitazione idrologica in Val Roja, Atti del Convegno VGR 2004 - Valutazione e Gestione del Rischio, Pisa, 19-21 Ottobre 2004.

Minciardi, R., Sacile, R., and Trasforini, E.: Assessing the efficiency and the criticality of the elements belonging to a complex territorial system subject to natural hazards, Nat. Hazards Earth Syst. Sci., 6, 21-32, doi:10.5194/nhess-6-21-2006, 2006.

Pascale, S., Giosa L., Sdao, F., and Sole, A.: Assessment of systemic vulnerability in flood prone areas, Fourth International Conference on Sustainable Development and Planning, Cyprus, 933-941, 13-15 May 2009.

Perrone, A., Iannuzzi, A., Lapenna, V., Lorenzo, P., Pisciteli, S., Rizzo, E., and Sdao, F.: High-resolution electrical imaging of the Varco d'Izzo earthflow (southern Italy), J. Appl. Geophys., 56, 17-29, 2004.

Polemio, M. and Sdao, F.: The role of rainfall in the landslide hazard: the case of the Avigliano urban area (Southern Apennines, Italy), Eng. Geol., 53, 297-309, 1999.

Polemio, M. and Sdao, F.: Rischio di frana indotto dagli eventi di pioggia in Avigliano (Basilicata), Geologia Applicata e Idrogeologia 31, 23-30, 1996 (in Italian).

Polemio, M. and Sdao, F.: Heavy rainfalls and extensive landslides occurred in Basilicata, Southern Italy, in 1976, in: Proceedings of 8th Congress of International Association of Engineering Geology and Environmen, Vancouver, Canada, Settembre 1998, edited by: Moore, D. and Hungr, O., Balkema (Ed.), Rotterdam, 3, 1849-1855, 5 ff., 2 tabb., 1998.

Schachter, R.: Probabilistic Inference and Influence Diagrams, Oper. Res., 36, 589-604, 1988.

Shenoy, P.: Valuation-Based systems for Bayesian Decision Analysis, Oper. Res., 40, 463-484, 1992.

Tamura, H., Yamamoto, K., Tomiyama, S., and Hatono, I.: Modeling and analysis of decision making problem for mitigating natural disaster risks, Eur. J. Oper. Res., 122, 461-468, 2000.

Thieken, A. H., Olschewski, A., and Kreibich, H.: Development and evaluation of FLEMOps - a new Flood Loss Estimation Model for the private sector, First International Conference on Flood Recovery, Innovation and Response, 315-324, 2008.

Varis, O.: Bayesian decision analysis for environmental and resource management, Environ. Modell. Softw., 12(2-3), 177-185, 1997.

Unesco - Undro: Natural disasters and vulnerability analysis, United Nations Disaster Relief Organization, New York, 386 pp., 1979. 